\title{
SEMI-COMPLETE PRIMARY ABELIAN GROUPS ${ }^{1}$
}

GEORGE KOLETTIS, JR.

1. Introduction. There are two kinds of primary abelian groups without elements of infinite height which can be described as being completely known. First, there are the direct sums of cyclic groups. Then there are those which, in the natural $p$-adic topology, coincide with the torsion subgroups of their completions. These are called torsion-complete [1, Exercise 58]. In each of these two cases the further knowledge of the Ulm invariants is sufficient to characterize the group.

It seems appropriate to combine the two and consider the class of groups obtained by taking the direct sum $H \oplus K$ of a torsion-complete group $H$ with a direct sum $K$ of cyclic groups. We call such a group semi-complete. While the representation $H \oplus K$ of a semi-complete group $G$ as a direct sum of a torsion-complete group and a direct sum of cyclic groups is not unique, some measure of uniqueness is present (Theorem 2), and any two such representations have isomorphic refinements (Theorem 3). These facts lead to an isomorphism theorem for semi-complete groups in terms of the Ulm invariants of $G, H$ and $K$ (Theorem 4).

As an application, in $\$ 4$ we use semi-complete groups to exhibit a continuum number of reduced primary abelian groups, each the power of the continuum, all with the same Ulm invariants, no two of which are isomorphic.

The material in this paper is essentially a portion of a doctoral dissertation submitted to the University of Chicago. The author wishes to thank Professor Irving Kaplansky for his constant advice and encouragement.

2. Let $G$ be a primary (always for a fixed prime $p$ ) abelian group without elements of infinite height, i.e., one for which $\bigcap_{n} p^{n} G=0$. The $p$-adic topology on $G$ is obtained by using the subgroups $p^{n} G$ as a base for the neighborhoods of 0 . Equivalently, $G$ is a normed group, the norm of a nonzero element $x$ being $2^{-h(x)}$. Here $h(x)$ denotes the height of $x$, the largest non-negative integer $n$ for which $x$ is in $p^{n} G$. If $H$ is a subgroup of $G$ and $H$ is pure $\left(p^{n} G \cap H=p^{n} H\right.$ for all $n$ ), the

Received by the editors June 12, 1959.

1 This paper was sponsored in part by the Office of Ordnance Research, United States Army, under Contract DA-11-022-ORD-1571 and by the Office of Naval Research. 
$p$-adic topology of $H$ is the same as the topology induced by that of $G$.

The torsion subgroup $G^{*}$ of the completion of $G$ is again a primary abelian group without elements of infinite height [1, Lemma 17], ${ }^{2}$ and $G$ is a pure subgroup of $G^{*}\left[1\right.$, Lemma 18]. $G^{*}$ is called the torsion-completion of $G$, and $G$ is torsion-complete in case $G=G^{*}$. Thus $G$ is torsion-complete if every Cauchy sequence $\left\{x_{i}\right\}$ of $G$, for which the orders of the elements $x_{i}$ are bounded, converges.

In any event, $G^{*} / G$ is divisible. For if $z$ is an element of $G^{*}$, the density of $G$ in $G^{*}$ gives an element $x$ in $G$ such that $z-x$ is in $p G^{*}$, which shows that $G^{*} / G=p G^{*} / G$.

Our first lemma shows that $G$ and $G^{*}$, while in general not isomorphic, have the same Ulm invariants.

Lemma 1. Let $G$ be a primary abelian group and $H$ a pure subgroup such that $G / H$ is divisible. Then the nth Ulm invariant $f_{G}(n)$ of $G$ equals the nth Ulm invariant $f_{H}(n)$ of $H$ for every finite ordinal $n$.

Proof. If $P$ denotes the subgroup of $G$ annihilated by $p$, the injection $H \rightarrow G$ induces a map

$$
\phi:\left(P \cap p^{n} H\right) /\left(P \cap p^{n+1} H\right) \rightarrow\left(P \cap p^{n} G\right) / P\left(\cap p^{n+1} G\right) .
$$

By the purity of $H,\left(P \cap p^{n+1} G\right) \cap\left(P \cap p^{n} H\right)=P \cap p^{n+1} H$ and so $\phi$ is injective. This shows that $f_{H}(n) \leqq f_{G}(n)$.

On the other hand, if $z$ is an element of $P \cap p^{n} G$, the divisibility of $G / H$ provides an element $x$ in $H$ (in fact, in $p^{n} H$ ) such that $z-x$ is in $p^{n+1} G$. Then $p(z-x)=-p x$ is in $p^{n+2} G \cap H=p^{n+2} H$. Say $-p x$ $=p^{n+2} y$ where $y$ is in $H$. The element $x+p^{n+1} y$ is in $P \cap p^{n} H$ and $z-x-p^{n+1} y$ is in $P \cap p^{n+1} G$. This shows that the coset $z+P \cap p^{n} G$ is the image of the coset $x+p^{n+1} y+P \cap p^{n} H$ and so $\phi$ is surjective. Thus $f_{H}(n)=f_{G}(n)$.

To obtain an explicit description of torsion-complete groups, one can appeal to a theorem of Fleischer and Kaplansky [1, Theorem 22]: every torsion-complete group $G^{*}$ is the torsion-completion of a direct sum of cyclic groups. In fact, $G^{*}$ is the torsion-completion of the subgroup $B$ generated by a maximal pure independent subset of $G^{*}$. $B$ is a direct sum of cyclic groups and $G^{*} / B$ is divisible. (Such a subgroup is called a basic subgroup [2, p. 181].) $B$ is unique up to isomorphism for $B$ is a direct sum of cyclic groups, and by Lemma 1 the Ulm invariants of $B$ are those of $G^{*}$. If we write $B=\sum B_{n}$, where $B_{n}$ is the direct sum of all the given cyclic summands of $B$ of order $p^{n}$,

${ }^{2}$ Only slight modifications are needed to adapt the results of $\$ 16$ in Kaplansky's book to the case we are considering. 
it is easy to see that the torsion-completion of $B$ is the torsion subgroup of the direct product $\prod B_{n}$. In particular, we see that a direct sum of cyclic groups is torsion-complete if and only if it is of bounded order.

3. Let $G$ be a semi-complete primary abelian group. For brevity, let us agree to call a decomposition $G=H \oplus K$ of $G$ standard if the first summand $H$ is torsion-complete and the second summand $K$ is a direct sum of cyclic groups. In general, $G$ will have many standard decompositions. For example, if $G=H \oplus K$ is a standard decomposition of $G$ and if $K=S \oplus K^{\prime}$ where $S$ is of bounded order, another standard decomposition of $G$ is $G=H^{\prime} \oplus K^{\prime}$ where $H^{\prime}=H \oplus S$; similarly for direct summands of bounded order of $H$. However, direct summands of unbounded order cannot be so shifted without destroying the standardness of the decomposition, and this is reflected in the following theorem.

THEOREM 1. Let $G=H \oplus K$ be a standard decomposition of the semicomplete group $G$, and let $T$ be a pure torsion-complete subgroup of $G$. Then there exists an integer $n$ such that $P \cap p^{n} T \subset H$.

Here $P$, as usual, denotes the subgroup of $G$ annihilated by $p$. We first prove two technical lemmas.

Lemma 2. Let $\left\{x_{i}\right\}$ be a sequence of elements of order $p$ in the primary abelian group $G$. If $h\left(x_{i}\right)=n_{i}$, say $x_{i}=p^{n_{i}} g_{i}$, where $n_{i} \neq n_{j}$ for $i \neq j$, then the subgroup $S$ generated by the $g_{i}{ }^{\prime}$ s is pure and is the direct sum of the cyclic groups generated by the $g_{i}$ 's.

Proof. If $S$ is not the direct sum of the cyclic groups generated by the $g_{i}$ 's, a relation $a_{1} x_{1}+\cdots+a_{s} x_{s}=0$, with not every $a_{i}$ a multiple of $p$, must hold. But this requires at least two of the $x_{i}$ 's to have the same height, contrary to hypothesis.

If $x$ is an element of order $p$ in $S, x$ is a linear combination of the $x_{i}$ 's by what we have just shown. Its height in $G$, and in $S$, is the smallest of the heights of the $x_{i}$ 's which appear nontrivially in the linear combination. Hence $S$ is pure [1, Lemma 7].

Lemma 3. Let $G$ and $G^{\prime}$ be primary abelian groups without elements of infinite height and $\pi$ be a homomorphism of $G$ into $G^{\prime}$ such that for all $n, \pi\left(P \cap p^{n} G\right) \neq 0$. Then there exists a pure subgroup $S$ of unbounded order of $G$, such that the restriction of $\pi$ to the closure $\bar{S}$ of $S$ is an isomorphism.

Proof. Using the fact that for all $n, \pi\left(P \cap p^{n} G\right) \neq 0$, we extract a sequence $\left\{x_{i}\right\}$ of elements of $P$ such that $x_{i}^{\prime}=\pi\left(x_{i}\right) \neq 0$ and such 
that $h\left(x_{i+1}\right)>h\left(x_{i}^{\prime}\right)$ for every $i$. Let $h\left(x_{i}\right)=n_{i}$ and $h\left(x_{i}^{\prime}\right)=n_{i}^{\prime}$. We

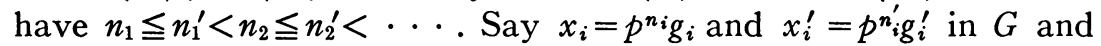
$G^{\prime}$, respectively. ( $g_{i}^{\prime}$ will not, in general, be the image of $g_{i}$.) Let $S$ and $S^{\prime}$ be the subgroups of $G$ and $G^{\prime}$ generated by the $g_{i}$ 's and by the $g_{i}^{\prime}$ 's. By Lemma $2, S$ and $S^{\prime}$ are pure in $G$ and $G^{\prime}$ and are the direct sums of the cyclic groups generated by the $g_{i}$ 's and the $g_{i}^{\prime}$ 's. $\pi$ induces an isomorphism between $P \cap S$ and $P^{\prime} \cap S^{\prime}$, and thus the restriction of $\pi$ to $S$ is an isomorphism. (The image of $S$ will not, in general, lie in $S^{\prime} ; P^{\prime}$ denotes the subgroup of $G^{\prime}$ annihilated by $p$.)

We note that in $G^{\prime}, p^{n_{i}^{\prime}} S^{\prime} \cap P^{\prime}=p^{n_{i}} \pi(S) \cap P^{\prime}$, for both are generated by the elements $x_{j}^{\prime}$ for $j \geqq i$.

Now let $y$ be an element of order $p$ in $\bar{S}$ for which $\pi(y)=0$. Say $y_{i} \rightarrow y$ where $y_{i}$ is in $S$. We can suppose that $y-y_{i}$ is in $p^{n_{i}^{\prime} G}$ for every $i$. A simple argument shows that, since $S$ is pure, each $y_{i}$ may be assumed to be of order $p$. Thus $-\pi\left(y_{i}\right)=\pi\left(y-y_{i}\right)$ is in $p^{n_{i}^{\prime}} G^{\prime} \cap S^{\prime} \cap P^{\prime}$ which is $p^{n_{i}} \pi(S) \cap P^{\prime}$. Since $\pi$ on $S$ is an isomorphism, this implies that $y_{i}$ is in $p^{n_{i}} S$. This proves that $y=0$.

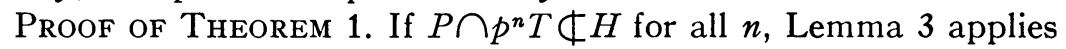
to show that there exists a pure subgroup $S$ of unbounded order in $T$ such that the projection $\pi: G \rightarrow K$ is an isomorphism when restricted to the closure $\bar{S}$ of $S$. Now $\bar{S}$ is pure [1, Lemma 20], and so the $p$-adic topology on $\bar{S}$ is the same as that induced by that of $T$. This means that $\bar{S}$ is torsion-complete. But $\bar{S}$ is isomorphic to a subgroup of $K$ and every subgroup of $K$ is a direct sum of cyclic groups by a theorem of Kulikov [1, Theorem 13]. We conclude that $\bar{S}$ is of bounded order, a contradiction.

Examples show that the stronger assertion, $p^{n} T \subset H$ for some $n$, is not true in general.

With Theorem 1 at hand we proceed to establish a relationship between two standard decompositions of a semi-complete group.

Theorem 2. Let $G=H \oplus K$ and $G=H^{\prime} \oplus K^{\prime}$ be standard decompositions of the semi-complete group $G$. There exists an integer $n$ such that $p^{n} H \cong p^{n} H^{\prime}$ and $p^{n} K \cong p^{n} K^{\prime}$.

Proof. Applying Theorem 1 twice, we deduce that for some nonnegative integer $n$, we have $P \cap p^{n} H=P \cap p^{n} H^{\prime}$. Hence $P \cap p^{n+i} H$ $=P \cap p^{n+i} H^{\prime}$ for every non-negative $i$. This implies that the Ulm invariants of the torsion-complete groups $p^{n} H$ and $p^{n} H^{\prime}$ are equal, so that $p^{n} H \cong p^{n} H^{\prime}$.

To show that $p^{n} K$ and $p^{n} K^{\prime}$ are isomorphic, we will show that $\left(P \cap p^{n+i} K\right) /\left(P \cap p^{n+i+1} K\right)$ and $\left(P \cap p^{n+i} K^{\prime}\right) /\left(P \cap p^{n+i+1} K^{\prime}\right)$ are isomorphic. It will follow that $p^{n} K$ and $p^{n} K^{\prime}$, as direct sums of cyclic 
groups with the same Ulm invariants, are isomorphic.

From $G=H \oplus K$, we have $P \cap p^{n+i} G=P \cap p^{n+i} H \oplus P \cap p^{n+i} K$. The kernel of the map $P \cap p^{n+i} G \rightarrow\left(P \cap p^{n+i} K\right) /\left(P \cap p^{n+i+1} K\right)$ is $P \cap p^{n+i} H$ $+P \cap p^{n+i+1} G$. Thus $\left(P \cap p^{n+i} G\right) /\left(P \cap p^{n+i} H+P \cap p^{n+i+1} G\right)$ and $\left(P \cap p^{n+i} K\right) /\left(P \cap p^{n+i+1} K\right)$ are isomorphic. Repeating the argument for $G=H^{\prime} \oplus K^{\prime}$, we obtain a similar isomorphism with $H$ and $K$ replaced by $H^{\prime}$ and $K^{\prime}$. Since $P \cap p^{n+i} H=P \cap p^{n+i} H^{\prime}$, the desired isomorphism is evident.

In the course of the proof, we saw that $P \cap p^{n} H=P \cap p^{n} H^{\prime}$ for a suitable $n$, so that, in this sense, $P \cap H$ is unique. It is easy to see that the corresponding assertion for $K$ and $K^{\prime}$ is false and that it is possible to have $K \cap K^{\prime}=0$.

Theorem 2 lends itself to showing that any two standard decompositions $G=H \oplus K$ and $G=H^{\prime} \oplus K^{\prime}$ of a semi-complete group have isomorphic refinements. We write $K=\sum K_{i}$ and $K^{\prime}=\sum K_{i}^{\prime}$ where every $K_{i}$ and $K_{i}^{\prime}$ is a direct sum of cyclic groups of order $p^{i}$. Also let $H$ and $H^{\prime}$ be the torsion subgroups of $\prod H_{i}$ and $\prod H_{i}^{\prime}$ where every $H_{i}$ and $H_{i}^{\prime}$ is also a direct sum of cyclic groups of order $p^{i}$. For every $i, H_{i} \oplus K_{i}$ and $H_{i}^{\prime} \oplus K_{i}^{\prime}$ are isomorphic for each is the direct sum of $f_{G}(i-1)$ copies of the cyclic group of order $p^{i}$. In addition, if $n$ is as in Theorem $2, H_{i} \cong H_{i}^{\prime}$ and $K_{i} \cong K_{i}^{\prime}$ for $i \geqq n$. This leads to the following theorem.

Theorem 3. Any two standard decompositions $G=H \oplus K$ and $G=H^{\prime} \oplus K^{\prime}$ of a semi-compete group $G$ have isomorphic refinements. Specifically, subgroups $\widetilde{H}, \widetilde{H}^{\prime}, \widetilde{K}$, and $\widetilde{K}^{\prime}$ of $H, H^{\prime}, K$, and $K^{\prime}$ exist with $\widetilde{H}$ and $\widetilde{H}^{\prime}$ torsion-complete, so that $G=\widetilde{H} \oplus B \oplus \widetilde{K}$ and $G=\widetilde{H}^{\prime}$ $\oplus B^{\prime} \oplus \tilde{K}^{\prime}$ where $B$ and $B^{\prime}$ are of bounded order and $\tilde{H} \cong \tilde{H}^{\prime}, \widetilde{K} \cong \widetilde{K}^{\prime}$, $B \cong B^{\prime}$.

Proof. Take $\tilde{H}$ to be the torsion subgroup of the direct product $\prod H_{i}$ for $i \geqq n, \tilde{K}$ to be the direct sum $\sum K_{i}$ for $i \geqq n$, and $B$ to be the direct sum $\sum\left(H_{i} \oplus K_{i}\right)$ for $i<n$. Similarly for $\tilde{H}^{\prime}, \widetilde{K}^{\prime}$, and $B^{\prime}$.

4. Let $G$ and $G^{\prime}$ be semi-complete groups with the same Ulm invariants. Suppose $G=H \oplus K$ and $G^{\prime}=H^{\prime} \oplus K^{\prime}$ are standard decompositions. When are $G$ and $G^{\prime}$ isomorphic? The answer is given by the following corollary to the discussion above.

THEOREM 4. $G$ and $G^{\prime}$ are isomorphic if and only if there is an $n$ such that for every $i \geqq n$, the ith Ulm invariants of $H$ and $H^{\prime}$ are equal and the ith Ulm invariants of $K$ and $K^{\prime}$ are equal.

As an application of Theorem 4, we show that there exist at least 
a continuum number of nonisomorphic groups, each the power of the continuum, having the same Ulm invariants. We do this by determining the semi-complete groups $G=H \oplus K$ having for every $i$, an $i$ th Ulm invariant equal to 1 . Such a group is determined up to isomorphism by the knowledge of the set $S$ of non-negative integers $i$ for which the $i$ th Ulm invariant of $H$ is 1 . For every subset $S$ of the set $I$ of non-negative integers, denote by $G_{S}$ the semi-complete group obtained in this way. If $S$ and $\Sigma$ are two subsets of $I$, Theorem 4 tells us that $G_{S}$ and $G_{\Sigma}$ are isomorphic if and only if the symmetric difference $S \triangle \Sigma$ is finite. Further, $G_{S}$ is countable only if $S$ is finite, and is otherwise of the power of the continuum. This proves that there are a continuum number of these groups having the cardinality of the continuum.

\section{BIBLIOGRA PHY}

1. I. Kaplansky, Infinite Abelian groups, Ann Arbor, University of Michigan Press, 1954. 1955.

2. A. G. Kurosh, The theory of groups, vol. I, New York, Chelsea Publishing Co.,

University of Notre DAME 